\title{
Not all views are equal, but all views matter
}

\author{
In cases of direct replications or direct critiques of earlier work, feedback from the original authors can have \\ an important role to play in the evaluation process, but such feedback is by definition not impartial. Our signed \\ comments policy allows such feedback to be incorporated in the consideration process, without impacting the \\ objectivity of peer review and editorial evaluation.
}

T: he peer review process must be based on feedback that is objective, impartial and free from conflicts of interest. However, there are instances where feedback from experts with the strongest possible conflict of interest can be useful to ensure that the work under consideration is accurate and robust. These rare instances involve research that replicates, directly challenges or directly builds on earlier work. They also involve non-research content (e.g., Perspectives, Comments and Reviews) that directly criticizes earlier work.

The authors of work that is subject to replication or challenge inevitably have an intellectual conflict of interest. It is natural to be invested in one's published work, to want to defend it against criticism and to look kindly on work that confirms one's own earlier conclusions. However, while conflicted, the authors of such work also likely have the most in-depth and detailed understanding of the technical aspects and content of their work.

Incorporating feedback from experts with this kind of intellectual conflict of interest poses a significant challenge to the evaluation process: for obvious reasons, it cannot form the basis for editorial decisions, but at the same time it can have a valuable role in ensuring that the work under consideration is valid and accurate. To resolve this challenge, we solicit signed comments from experts with a vested interest in the work under consideration (either negative or positive), where the conflict of interest is declared and whose function in the consideration process is distinct from the feedback provided by independent reviewers.

We have now formalized our policy for signed comments, which can be found on our website (https://www.nature.com/ nathumbehav/signed-comments). Here, we highlight important aspects of this policy.
Signed comments are not peer reviews. Our decisions on whether to reject a manuscript or invite a revision are based exclusively on the feedback provided by our independent reviewers. However, the authors of signed comments have direct and in-depth knowledge of the methods and analytical approach in their previously published work, and they may be in a position to raise methodological issues that are not raised by any of our independent reviewers. If we judge these methodological issues to be potentially significant and valid, we will share the signed comments with one or more of our independent reviewers, asking for their feedback. If our independent reviewers corroborate the validity of the methodological concerns, these concerns can then inform the editorial decision.

The authors of signed comments are asked to refrain from providing qualitative evaluations of the work or recommendations for or against publication of the work, as these will be ignored. Instead their feedback must be limited strictly to methodological issues and to the accuracy with which their work is represented in the target manuscript. If the authors of signed comments provide feedback that goes beyond these points, their comments will be returned to them for revision or will be editorially redacted.

It is also important that, upon invitation, the authors of signed comments provide their feedback promptly, within the specified timeframe. We will not delay decision-making for signed comments if all our independent reviewers have returned their reports and the deadline for submission of signed comments has passed. Prompt engagement is essential to ensure fairness towards the authors of the work under consideration.

We do not accept comments from authors with a conflict of interest if they are unwilling to sign them. For instance, if a piece of research we are considering replicates an earlier study, and the authors of that study provide comments on the current work, but do not agree to sign them, the comments will be suppressed and not shared with the authors and our other reviewers. Only reviews provided by independent peers who are free from conflicts can be unsigned (to prevent timid reviews, reduce opportunities for favour trading and correct for power imbalances).

Although signed comments do not form the basis for editorial decisions, if we invite a revision, comments provided by the authors of the original work can inform our requests for revisions, and we ask that our authors provide point-by-point responses to these comments in the same manner as they respond to the feedback of our independent reviewers.

As of 1 December 2019, we have implemented transparent peer review for authors who opt in. Signed comments, although they do not constitute peer reviews, do form part of the review record. Authors of signed comments need to be aware that, if the authors of the manuscript opt in to transparent peer review, their signed comments will be published along with the manuscript.

Scientists disagree (often quite fundamentally), criticize each other's work and question each other's findings. This is all as it should be. When these disagreements and critiques come from a place of competing interests, however, they are in direct conflict with the aims of the peer review process. By developing a distinct, clearly circumscribed and transparent role for such feedback in the evaluation process, we believe that the work we consider can only become more robust.

Published online: 16 July 2020 https://doi.org/10.1038/s41562-020-0923-9 